Diplomstudiengang Ökonomie mit ökologischem Schwerpunkt

\section{Interdisziplinarität als Ziel}

\section{An der Carl-von-Ossietzky-Universität Osnabrück wird derzeit an der Konzeption eines Diplomstudienganges Ökonomie mit ökologischem Schwer- punkt gearbeitet.}

]

Von Reinhard Pfriem

$\mathrm{n}$ diesem Informationsdienst sind in den ver-

gangenen Jahren einige Berichte erschienen, die Fortschritte dokumentierten im wirtschaftswissenschaftlichen Hauptstudium an den Universitäten oder auch an den Fachhochschulen ökologische Schwerpunkte zu setzen. Dies betraf Hauptstudiumsschwerpunkte auf dem Weg zum traditionellen betriebs- bzw. volkswirtschaftichen Examen (oder, nicht nur bei den Gesamthochschulen, zum integrierten Examen des DiplomÖkonomen). Es tastete aber den in der Regel „ökologiefreien“ Kanon der Ausbildungsinhalte des Grundstudiums nicht an, sodaß weiterhin der Satz gilt: man kann an jeder deutschen Universität ein betriebswirtschaftliches Examen machen, ohne die ökologische Dimension wirtschaftlichen Handelns auch nur in einer einzigen Lehrveranstaltung zur Kenntnis nehmen zu müssen.

\section{- Ausgangssituation der Fachbereiche}

An der Carl-von-Ossietzky-Universität Oldenburg ist gegenwärtig das Bemühen im Gange, die Verankerung ökologischer Inhalte im wirtschaftswissenschaftlichen Studium in beiden Punkten (Studienabschluß und allgemeines Grundstudium) etwas kräftiger anzugehen. Dabei kommen uns einige in jüngerer Zeit bewußt veränderte Bedingungen zugute:

der Fachbereichsrat hat nach entsprechenden Vorarbeiten einer auf Zeit eingesetzten Strukturkommission unter anderem beschlossen, die ökologische Orientierung $\mathrm{zu}$ einem wesentlichen Baustein der Fachbereichsentwicklung nach innen und außen zu machen;

- im Fach Betriebswirtschaftslehre existiert seit dem 1. April 1994, faktisch schon einige Semester länger, ein Lehrstuhl für Allgemeine Betriebswirtschaftslehre, Unternehmensfïhrung und betriebliche Umweltpolitik (Pfriem, Hallay) und wird aktuell ein weiterer Lehrstuhl mit der Denomination Produktion und Umwelt ausgeschrieben; Wolf, jetzt Meyerholt); von Studierendeninteressen bei. wirtschaftswissenschaftlichen Studiengang nachzudenken. Insofern lag es nahe, daß der Fachbereichsrat eine Arbeitsgruppe einsetzte mit dem Auftrag, die konkrete Konzeption für einen solchen neuen Diplomstudiengang zu entwickeln. Diese Arbeitsgruppe hat inzwischen ihre Arbeit abgeschlossen, die Konzeption wird derzeit in der ständigen Studienkommission des Fachbereichs sowie im Fachbereichsrat selbst abschließend beraten reits seit einer Reihe von Jahren zwei Lehrstiihle mit umweltpolitischer Schwerpunktsetzung in Lehre und Forschung (Pfaffenberger, Ströbele), was u.a. zu einem Graduiertenkolleg Umweltund Ressourcenökonomik beitrug - nach dem Wechsel des Kollegen Ströbele nach Münster wurde diese Stelle bereits im Dezember 1995 mit einschlägigen Absichten neu ausgeschrieben;

im Fach Recht, das in Oldenburg in den wirtschaftswissenschaftlichen Fachbereich integriert ist (neben den Studiengängen für Lehramt und Magister gibt es bisher zwei Diplomstudiengänge, nämlich Wirtschaftswissenschaften und Betriebswirtschaftslehre mit juristischem Schwerpunkt), ist die ökologische Spezialisierung auf Mittelbauebene im Rahmen des Lehrstuhls für öffentliches Recht vertreten (vormals

- ebenfalls auf Mittelbauebene gibt es eine ökologische Spezialisierung auch im Lehramtsbereich für berufsbildende Schulen (Siehlmann);

- am Fachbereich besteht mit der IGUWi (Interessengemeinschaft Umwelt und Wirtschaft) seit einigen Jahren eine Studierendeninitiative, die sich mit entsprechenden inhaltlichen und didaktischen Themen beschäftigen. Ein dort initiiertes interdisziplinäres Projekt „Ökologischer Strukturwandel in der Nord-West Region“ kann als Vorlauf zur Erprobung der Kooperation der verschiedenen Fachbereiche dienen. Darüber hinaus trugen Gespräche mit Mitgliedern der IGUWi zur Berücksichtigung

Bereits mit diesen internen Entwicklungen des Oldenburger Fachbereichs Wirtschafts- und Rechtswissenschaften waren bzw. sind hervorragende Bedingungen dafür gegeben, in der Lehre mehr zu machen als ein oder zwei Wahlpflichtfächer anzubieten. Diese Entwicklung zeichnete sich bereits ab, als in den ersten Monaten des vergangenen Jahres von der Leitung der in anderen Fachbereichen seit längerem auf Ökologie großes Gewicht legenden Universität positive Signale kamen, über einen entsprechenden

\section{- Konzeption des neuen Studiengangs}

Angesichts des bereits artikulierten fachbereichsinternen Einvernehmens in allen wichtigen Teilfragen und der zu erwartenden Zustimmung der Universitätsleitung sowie des niedersächsischen Wissenschaftsministeriums (zumal wir mit der Implementation dieses Studiengangs keine zusätzlichen Stellenforderungen verbinden) sollen im folgenden einige Grundelemente der Konzeption vorgestellt werden.

1. Der Studiengang soll beiden fachlichen Dimensionen hinreichend gerecht werden. Das bedeutet: Der Studiengang ist ein ökonomischer, die wirtschaftswissenschaftliche Ausbildung muß hinreichend gründlich sein. Und umgekehrt werden erstmalig in Deutschland nicht nur umweltbezogene Schwerpunkte im Rahmen des wirtschaftswissenschaftlichen Studiums gesetzt, sondern wird ein eigenes Examen geschaffen. Die ökologischen Ausbildungsinhalte müssen also entsprechend gründlich und ausführlich sein, damit auch von der ökologischen Seite keine berechtigte Kritik geführt werden kann, die Bezeichnung des Studiengangs sei mehr Verpackung als Inhalt.

2. Die für einen Diplomstudiengang Ökonomie mit ökologischem Schwerpunkt erforderliche Interdisziplinarität kann in Oldenburg durch die Verknüpfung des fachbereichseigenen Lehrpotentials mit je einem Kollegen aus dem naturund dem sozialwissenschaftlichen Bereich hervorragend hergestellt werden: Prof. Dr. Dieter Schuller (Institut für Ökochemie und Umweltanalytik) sowie Prof. Dr. Horst Zilleßen (Schwerpunkt Umweltmediation und -konfliktmanagement).

3. Als Kernstiick des neuen Studienganges soll es auf dieser Grundlage einen Pflichtblock ökologische Ökonomie im Hauptstudium geben, der 20 Semesterwochenstunden (SWS) umfaßt, je vier SWS betriebswirtschaftliche, volkswirt- 
schaftliche, rechtliche, naturwissenschaftliche und sozialwissenschaftliche Behandlung des ökologischen Problemfeldes. Dafür werden z.B. aus dem naturwissenschaftlichen Bereich Veranstaltungen angeboten zu den Themen ,Wasser - Stoff zum Leben - Lebensraum Gewässer" und „Technische Verfahren zum Schutz der Umwelt". 4. Aus diesen fünf Fächern sollen die Studierenden ein Vertiefungsfach wählen, in dem sie über die vier SWS hinaus weitere acht $\mathrm{zu}$ absolvieren und nicht nur eine einfache, sondern eine doppelte Prüfungsleistung zu erbringen haben (Vorund Hauptleistung). Beispielsweise sollen für die naturwissenschaftliche Vertiefung zwei zweistiundige Veranstaltungen zu den Themen ,Die Atmosphäre. Entstehung, Entwicklung, Prozesse (Wetter, Klima), Gefährdung“ sowie „Sedimente und Böden - die Registratur der Sünden - Altlasten und ihre Bewältigung“ bereitgestellt werden, ferner ein Praktikum „Umweltanalytischer Grundkurs“ und/oder ein Betriebspraktikum in der Arbeitsgruppe Ökochemie und Umweltanalytik.

5. Auf der anderen Seite dieses ökologiebezogenen Hauptstudiumteils verbleiben je vier SWS allgemeine Betriebs- und Volkswirtschaftslehre sowie zwei weitere Wahlpflichtfächer von zweimal zwölf SWS, wovon eines entweder aus der Betriebs- oder Volkswirtschaftslehre gewählt werden muß. Damit ist jenseits der ökologischen Ausbildungsinhalte ein genügend gründliches allgemeines wirtschaftswissenschaftliches Fundament für die Examensqualität gegeben.

6. Für die Konzeption des künftigen Grundstudiums ist von Belang, daß - ebenfalls ohne zusätzliche Stellen und hier in Kooperation mit dem Fachbereich Informatik - parallel auch ein Studiengang Wirtschaftsinformatik eingerichtet werden soll. Die wesentliche Idee für das Grundstudium heißt nun, daß es auf alle vier Diplomstudiengänge einfuihrend vorbereiten soll. Ökologische Lehrinhalte werden dementsprechend bereits in das Grundstudium integriert und gehören damit zum Pflichtkanon auch für alle diejenigen Studierenden, die sich für einen anderen Diplomstudiengang als den mit ökologischem Schwerpunkt entscheiden. Konkret werden vier SWS des Grundstudiums inklusive verpflichtender Prüfungsleistung dem Ökologiebezug gewidmet werden. Weil die Rechtswissenschaft im Oldenburger Grundstudium aufgrund der konkreten Fachbereichskonstruktion bereits eine sehr starke Stellung einnimmt, werden diese vier SWS pragmatischerweise von den anderen vier Fächern getragen.

\section{Der Autor}

Reinhard Pfriem ist Professor der Betriebswirtschaffslehre an der Universität in Osnabrück.

Kontakt: Reinhard Pfriem, Carl-von-OssietzkyUniversität Oldenburg, Institut BWLI, Postfach 2503,26111 Oldenburg. Tel. (0441) 798-8345 (Sekr. -8356)

Nach Absprache der betreffenden Kollegen soll während eines Zyklus eine gemeinsame Problemstellung behandelt werden (z.B. nachhaltige Entwicklung). Selbstverständlich wird darauf geachtet, daß diejenigen Studierenden, die nach dem Grundstudium Oldenburg verlassen wollen, keine Akzeptanzprobleme an anderen Universitäten bekommen.

7. Natürlich kann es nicht die Funktion der Einführung eines neuen Studienganges sein, die überfälligen didaktischen, prüfungstechnischen und berufsbildbezogenen allgemein erforderlichen Studienreformen im Sinne von Gratiseffekten gleich mitzuerledigen. Gleichwohl lehren die praktischen Erfahrungen im Bereich ökologischer Unternehmenspolitik und verwandten Feldern, daß die universitäre Ausbildung mehr als bisher üblich (leider auch in Oldenburg) die sozialen, kommunikativen und organisatorischen Kompetenzen der Studierenden stärken muß. Das erfordert seitens der Lehrenden, die jeweils existierenden Studien- und Prïfungsordnungen maximal in dieser Richtung auszunutzen bzw. verändern zu helfen. Wer sich im Unternehmen bewähren will, wird dies nicht hauptsächlich durch Klausurenschreiben schaffen...

Einen konkreten Ansatz in dieser Richtung versuchen wir mit der Festlegung, daß im gewählten ökologischen Vertiefungsfach entweder der Vor- oder der Hauptleistungsschein erworben werden soll durch eine

kommunikative Leistung (Arbeitsgruppe leiten, Tutorium, Studierendenübung von einem Semester), oder eine

organisatorische Leistung (Organisierung von Veranstaltung(en) oder Exkursion(en) oder eine

- kooperative Leistung (Auftun einer Mentorfirma, Praktikum unter bestimmten Fragestellungen mit Praktikumsbericht).

Kommunikation und Kooperation gelten natürlich auch für uns selber: Wir freuen uns natürlich über Anregungen, Kritik und Kontaktaufnahme.

Zur Notwendigkeit von gemeinsamen wissenschaftlichen Anstrengungen

\section{Forschungskooperation für eine global nachhaltige Entwicklung}

\section{Der Autor skizziert die Grundlage für eine gemeinsame Forschung und weitere Aktivitäten für eine global nachhaltige Entwicklung. Ein Aufruf zu einer inter- disziplinäre Forschungskooperation.}

$\mathcal{1}^{\circ}$ ber Nachhaltige Entwicklung gibt es bisher keine einheitliche Vorstellung. Dies dürfte angesichts der unterschiedlichen Ausgangsbedingungen in den verschiedenen Weltregionen und angesichts divergierender Interessen sozialer Gruppen sowie als Folge unterschiedlicher ethischer Orientierungen auch in Zukunft kaum erreichbar sein. Die Spannweite der Konzepte der nachhaltigen Entwicklung reichen vom Konzept des nachhaltigen Wirtschaftswachstums bis zu Nachhaltigkeitsvorstellungen, die jedweden Eingriff in die globalen Ökosysteme ausschließen. Die erste extreme
Position ist eine Umschreibung der in eine Krise geratenen Modernisierungsstrategie und die letzte Extreme stellt das Existenzrecht selbst der gegenwärtigen Weltbevölkerung von rund fünf Milliarden radikal in Frage.

Anzustreben ist eine global nachhaltige Strategie, die ökonomische, ökologische, soziale, politische und kulturelle Dimensionen einschließt. Diese ganzheitliche Entwicklungsstrategie ist zweifelsohne weitreichender als die eindimensional auf ökonomisches Wachstum orientierten Modernisierungskonzepte. Gleichwohl bedeutet sie sowohl für die Wissenschaft als auch für die Praxis unvergleichbar größere 
(c) 20I0 Authors; licensee IÖW and oekom verlag. This is an article distributed under the terms of the Creative Commons Attribution Non-Commercial No Derivates License (http://creativecommons.org/licenses/by-nc-nd/3.o/), which permits unrestricted use, distribution, and reproduction in any medium, provided the original work is properly cited. 